\title{
Justice Beyond Utility in Artificial Intelligence
}

\author{
Lily $\mathrm{Hu}$ \\ Harvard University \\ Cambridge, MA \\ lilyhu@g.harvard.edu
}

\begin{abstract}
The entry of Artificial Intelligence into prominent social and economic environments has brought to the fore concerns about the ethical nature of such agents and tools. Though it is well-known that methods based in utilitarian calculus often fail to account for moral considerations, few alternatives have been adopted in the field of AI. My work advocates for a new approach toward the interaction between AI methods and the social that centers principles of distributive justice. As AI increasingly drives consequential social decision-making, we must consider not only what can be done but what ought to be done. Grappling with the inherently normative nature of these problems requires an orientation towards $\mathrm{AI}$ that is able to conceptualize justice beyond utility.
\end{abstract}

\section{ACM Reference Format:}

Lily Hu. 2018. Justice Beyond Utility in Artificial Intelligence. In 2018 AAAI/ACM Conference on AI, Ethics, and Society (AIES'18), February 2-3, 2018, New Orleans, LA, USA. ACM, New York, NY, USA, 2 pages. https: //doi.org/10.1145/3278721.3278798

In our field of Artificial Intelligence, the machine-behavioral realization of models based in neoclassical economics and utilitarian calculus represents not only a successful test-of-concept, but we may view such principles as in fact, one of the first concrete and systematic operationalizations of an ethical theory in the "real world." Here, a notable paradox stands: While the ethics of the field of AI at-large is under continual debate and contention, the conversation about the ethical grounding of individual AIs in particular is rather uncontroversial-AI tools, whether it be a reinforcement learning agent or a machine learning-based classifier, are for the most part, act utilitarians-that is, the right action is the one that maximizes (expected) utility.

When the reach of AI was largely limited to solving technical tasks, researchers could view the dictates of utility maximization as a purely procedural approach to determining agent action. But the entry of AIs into the realm of the social has forced a shift in our evaluation of the rightness of utility-based models, resurfacing the fundamentally ethical nature of agent decision-making. While philosophers have known for quite a while that utilitarianism alone as a basis for all action can give an impoverished account of justice or fairness, the realization has been a recent rude awakening for computer scientists working in Artificial Intelligence and Machine Learning who are now newly grappling with issues of injustice that

Permission to make digital or hard copies of part or all of this work for personal or classroom use is granted without fee provided that copies are not made or distributed for profit or commercial advantage and that copies bear this notice and the full citation on the first page. Copyrights for third-party components of this work must be honored.

For all other uses, contact the owner/author(s).

AIES '18, February 2-3, 2018, New Orleans, LA, USA

(c) 2018 Copyright held by the owner/author(s).

ACM ISBN 978-1-4503-6012-8/18/02.

https://doi.org/10.1145/3278721.3278798 arise when automated utility-maximizing tools are at the helm of social decision-making processes. Cathy O'Neil's Weapons of Math Destruction [6] and ProPublica's audit of the COMPAS recidivism tool [1] are just two of a series of high-publicity investigations that have uncovered the surprising fact that seemingly objective and purely optimization-driven devices can exhibit human-like biases in socially-oriented tasks, in many cases producing behaviors that are considered abhorrent and even unlawful.

But within the utility-maximizing model of AIs, encouraging behaviors and outcomes that align with social values tends to be of secondary interest. My work examines the extent to which the governing ethos of utility-based rationality built into AI systems is compatible with societal interests and norms of fairness. In particular, when AI techniques are employed as resource allocation mechanisms-whether it be sifting through job candidate résumés to offer interview slots or defendant data to produce recidivism risk scores-unconstrained maximization of predictive accuracy as utility has been shown to reinforce and deepen racial, gender, and class inequalities. As such, the demands of fairness must coexist alongside or be built into the existing utilitarian framework of AI systems. Thus my work asks: How can the variable demands of justice as fairness be computationalized, so as to fit within a utility-based AI system, in a way that approximates the dynamic environment of the social world?

Research in the growing literature of algorithmic fairness has studied similar questions by beginning with a domain-general "definition" of fairness and then constraining the behavior of particular algorithms so to align with the fairness notion presented. However, the problem of generating general principles of fairness is not only a notoriously difficult task in itself, but such an approach lacks the context to handle the particular trespasses of justice at stake in domains with distinct histories, patterns of inequality, and moral obligations. I claim that we cannot adequately evaluate the social and ethical impact of an algorithm's behavior without examining deeply the particular system within which it is embedded. Since AIs rarely fully control a resource distribution process, my work models and analyzes the dynamics of an algorithm's whole system of use to determine what type of intervention would be appropriate to achieve an outcome that can be argued to be just for a particular system.

In work presented at the World Wide Web Conference (WWW 2018), I tackle the problem of algorithmic reinforcement of disparate group outcomes in the labor market [3] and argue that relying on leading notions of algorithmic fairness to constrain hiring practices are insufficient to overcome the steeped inequalities that characterize every cut of the employment cycle. I prove that when the group-memberships of job candidates are observable, such as race and gender, and decision-makers are equipped with standard homo economicus capabilities such as Bayesian reasoning, conceptions of 
individual and meritocratic fairness, which constrain algorithms to treat similarly qualified people similarly $[2,4]$, continue to foreground short-term utility maximization, justifying disparate outcomes in a vicious cycle that fails to achieve long-term societal goals of ensuring equality of opportunity.

A central argument of my research contends that these static utility-based conception of optimal hiring, wherein algorithms predict and hire the "good" workers out of a candidate pool, is ill-suited for understanding the dynamics of complex social processes and as a result, the societal obligations to which AI tools may be bound. Instead, my work widens the view of algorithmic fairness to consider the dynamics of the entire labor market system, from workers' investment opportunities prior to entering the labor market to their tenure within the market as they interact with various firms and cycle through different jobs. In re-embedding algorithms in their social and human contexts, my work preserves aspects of rational choice theory that bear on human behavior while departing from a popular machine learning practice of treating human data as $a$ priori parameters of a utility function rather than the products of structurally influenced human actions. My stylized model casts a worker as a rational actor navigating a sequence of stages wherein she has attributes both personal (such as ability level) as well as social (such as group membership), faces individualized education investment costs, and makes employment-related decisions. Labor market interactions between workers and hiring-agents are embedded within a reputational dynamic repeated game where changing group reputations, which approximate societal standing, bear on members' investment costs-for two workers equal in innate ability, a lower reputation group member faces higher costs-as well as a hiring agent's perception of the worker's qualifications.

When initial group reputations are unequal, worker and firm best responses may cause the system to converge to an asymmetric equilibrium with disparate outcomes. Further, I prove that this reputation system has feedback and externality properties such that even when standard algorithmic fairness definitions are in place, the asymmetric equilibrium in which workers of the same ability level but of different groups face disparate wage prospects is maintained.

As evidenced by the methodology of this work, I do not dispose of the concepts of rationality and utility, rather I borrow techniques from both economics and sociology to build a model of the labor market pipeline that is better able to pinpoint an underlying origin of algorithmic disparate outcomes, shifting from a data-centric to an action-centric view of the world. For the final upshot of this work, I designed a fairness intervention on hiring practices to address the empirically-validated social phenomenon of development bias, in which members of a disadvantaged group are disproportionately excluded from opportunities required to realize their goals, a leading source of disparate employment outcomes [5]. I prove that my proposed short-term intervention installs long-term social fairness by converging the system to a group-equitable steady-state, and that moreover, under weak market conditions, the "fair" equilbrium outcome Pareto-dominates the asymmetric steady-state arising under unconstrained or procedurally fair hiring.

My paper on fair hiring in the labor market is one of the first works in the algorithmic fairness community that explicitly models the impact of algorithms in situ and makes a comparative statics social welfare argument against existing propositions of fairness. I also developed an argument grounded in legal and philosophical discourse for the ethicality of both the intervention proposed and the final group-egalitarian outcome in the labor market that is not based in utilitarian calculus. My inclusion of such content is rare in the fairness literature and highlights the central role that I believe ethics and justice must play in computer science and mathematical research on algorithmic biases.

My current research constructs a mathematical relationship between the problem of utility maximization with fairness constraints devised by AI researchers and the problem of designing social welfare functionals that embed distributive principles that is considered in welfare economics and social choice theory. By drawing a connection between the two approaches, I aim to also bring to light the latter scholarship's tradition of fitting normative analysis and argument alongside mathematical model construction. In separate work of a more critical nature, I argue that the staunch commitment to a methodology of utility optimization leads to a fallacy of choice among "fairness definitions." By delimiting the problem of fairness in AI systems to tinkering with the nuts-and-bolts decision criteria alone, research has implicitly assumed that fair algorithms can operate self-sufficiently without reference to humans or context. Not only is this "set-and-forget" tactic ill-suited to most social realms, but it also has the effect of blinding practitioners to the possibility for more holisitc ethical design of AI decision-making procedures and pipelines.

While the constructs of utility and rationality continue to be invaluable for AI systems grappling with societal values, it is also crucial that a conception of justice may exist as independent and distinct from any other utility maximization problem. My thesis sits at this region lying in between, at the intersection of utilitarianism as a framework and methodology of algorithm theory and justiceas-fairness as an ethical and social aspiration, characterizing aspects of this still under-explored landscape.

\section{REFERENCES}

[1] Julia Angwin, Jeff Larson, Surya Mattu, and Lauren Kirchner. 2016. Machine bias: There's software used across the country to predict future criminals. and itâĂŹs biased against blacks. ProPublica, May 23 (2016).

[2] Cynthia Dwork, Moritz Hardt, Toniann Pitassi, Omer Reingold, and Richard Zemel. 2012. Fairness through awareness. In Proceedings of the 3rd Innovations in Theoretical Computer Science Conference. ACM, 214-226.

[3] Lily Hu and Yiling Chen. 2018. A Short-term Intervention for Long-term Fairness in the Labor Market. In Proceedings of the 2018 World Wide Web Conference on World Wide Web. 1389-1398.

[4] Michael Kearns, Aaron Roth, and Zhiwei Steven Wu. 2017. Meritocratic fairness for cross-population selection. In International Conference on Machine Learning. $1828-1836$.

[5] Glenn C Loury. 2009. The anatomy of racial inequality. Harvard University Press.

[6] Cathy O'Neil. 2017. Weapons of math destruction: How big data increases inequality and threatens democracy. Broadway Books. 